\title{
Forecasting market demand for new telecommunications services: an introduction
}

\author{
Peter McBurney ${ }^{\mathrm{a}, *}$, Simon Parsons ${ }^{\mathrm{a}}$, Jeremy Green ${ }^{\mathrm{b}}$ \\ a Department of Computer Science, University of Liverpool, Liverpool L69 7ZF, UK \\ b Ovum Ltd., 12 Farringdon Road, London EC1M 3HS, UK
}

\begin{abstract}
The marketing team of a new telecommunications company is usually tasked with producing forecasts for diverse stakeholders with different needs. Consequently, those outside marketing often realize neither the many reasons for developing forecasts nor the marketing theory used and the challenges involved in doing so. Based on our three decades of experience working with telecommunications operators around the world we seek to redress this situation by presenting a discussion of the issues involved in demand forecasting for new communications services. (C) 2002 Elsevier Science Ltd. All rights reserved.
\end{abstract}

Keywords: Demand forecasting; New product marketing; Telecommunications services

\section{Introduction}

With the liberalization of telecommunications markets around the world and the development of new communications technologies, new services are being offered to potential customers at an increasing rate. Methods of forecasting demand which require historical data (such as time series analysis and econometric methods) cannot be used for entirely new services. This paper outlines some of the challenges involved in forecasting demand for new telecommunications services and describes the current best practice based upon the combined experiences - totalling almost 30 years - of

\footnotetext{
${ }^{*}$ Corresponding author.

E-mail addresses: p.j.mcburney@csc.liv.ac.uk (P. McBurney), s.d.parsons@csc.liv.ac.uk (S. Parsons), jmg@ovum.com (J. Green).
} 
the authors working and consulting for telecommunications operators in Europe, the Americas and Asia.

Typically, the department tasked with generating demand forecasts for the services to be provided by a new telecommunications company (telco) is the marketing department. Because of this, marketers are usually aware of the diverse nature of those requesting forecasts and, at least in general terms, their reasons for doing so. Similarly, having to produce the figures, marketing personnel are usually aware of the challenges and implicit assumptions involved in this task. However, in our experience, such awareness is rarely shared by other groups in the enterprise or those outside it. Many times, in our experience, a large gulf of understanding separates the marketing team from the engineering or finance departments over the reasons for forecasting and challenges involved in it. Part of this gulf arises from the different terminologies and concepts each group of professionals commonly use; it is still rare to hear telecom marketers speak of erlangs, for instance. Another reason for the gulf, in our opinion, is the diverse requirements of those using the forecasts, so that marketing teams often appear to others to be merely reacting to whichever is the latest pressure for higher, or sometimes lower, forecasts.

We hope that this paper can go some way to remedy this situation. We begin with a brief introduction to the relevant parts of the marketing theory, which provides the context within which demand forecasts are generated. We then identify, in Section 3, the key stakeholders in the forecasting process in a new telecommunications company, and their broad reasons for having an interest. While some or all of these will be well known to senior managers involved in start-up telcos, we have not seen this material presented before in print. It will be seen that the interests of stakeholders are diverse, and often conflicting. Section 4 outlines at a high level the main forecasting techniques in use for new telecommunications services, while Section 5 describes some of the conceptual and practical challenges involved in using these. The material in these two sections is well known to those involved in the practice of forecasting, but again is material we have not before seen consolidated in print. Our experience leads us to believe that people outside the marketing departments of telcos are not usually well-acquainted with these issues. We conclude in Section 6 with a brief discussion of the lessons for forecasters which the authors have acquired over the course of their careers. Throughout the paper, we use the words products and services interchangeably.

\section{Marketing strategy}

The key task of marketing strategy and implementation in the pre-launch period is to develop a marketing strategy so as to achieve the company's objectives in the marketplace (Hemming et al., 1996; Kotler, 1991; Urban and Hauser, 1993) This task can be summarized in three key questions: What are we going to sell? To whom? And, how? These might appear straightforward questions, but answering them 
sufficiently precisely for business implementation often requires considerable analysis and thought, including consideration of many subtle alternatives. Selecting between these alternatives almost always requires the making of trade-offs between options none of which is inherently better than the others.

As an example, imagine we are planning to sell high-capacity international telecommunications links. Are these to be sold to large corporate end-customers or to other telecommunications operators? These two groups of target customers are likely to have different requirements in terms of quality of service, redundancy, latency, etc., and different requirements for after-sales support and service. They are likely to have very different purchase decision processes and price expectations. They may have different expectations as to how they are to be sold to, how negotiations are to be conducted, and the durations of contracts. Indeed, there are also likely to be significant differences within each of these two groups on each of these dimensions, for example, due to the extent of competition each company faces in its own marketplace. Companies in the financial sector, for instance, are typically more demanding of telecommunications suppliers - both during purchasing negotiations and subsequently - than are customers in other industry sectors, due to the larger stakes and fiercer competition involved. Our marketing strategy needs to define which types of customers we will target, with what offers and in what manner.

Marketers typically describe marketing strategy development in terms of the factors over which the company, to a greater or lesser extent, has influence. These are often summarized as "The Four Ps" (Kotler, 1991), but for telecommunications services there is in fact a fifth, Permission. Each of these factors suggests a number of questions which company's marketers need to answer:

1. Product. What is the product or service and what are its specific features? Which features will comprise the core product, and which supplementary or value-added products? The marketing team may not have complete freedom in product design, due to technical, regulatory and financial constraints, but even within these, there is usually scope for considerable variations.

2. Price. What price structures and levels will be adopted? What discounts, if any, will be provided, when and to whom? What charges, if any, will be levied for additional services, billing options, after-sales support, or ancillary items (such as leather cases for mobile phones). What credit terms will be provided, and to whom? In most industries, companies freely decide the price structures and levels of the services they provide. In telecommunications, however, operators are often constrained by regulation, for example, having to obtain Government approvals before setting price levels. Even in relatively liberal telecommunications markets, such as the USA, operators may still have to file their proposed prices to regulatory agencies in advance of their date of effect. One definition of marketing is: all those things the company can do to sell its products without lowering its prices.

3. Promotion. How will the new service be advertised and promoted? What marketing communications material (brochures, posters, etc.) will be provided at retail 
points-of-sale and to sales staff? What messages to which audiences will be conveyed by each type of communication?

4. Place. Where and how will the service be sold? How will equipment (e.g., mobile handsets) be provided to the various points-of-sale? Where and how will customers be activated to the service? In what way will after-sales services be provided? How will equipment returns and replacement be effected? How will other entities in the service distribution channel be selected, managed and compensated?

5. Permission. Most telecommunications services in most countries are regulated, to a greater or lesser extent, by telecommunications-specific laws and agencies. For instance, the scarcity of radio frequency spectrum usually means that governments require intending operators of public wireless networks to have a licence to build and operate the network before providing public services. A new company therefore needs to obtain the necessary permits to offer services, and this may or may not be straightforward. For intending global companies, both international licences and national licences in many countries may be required. Regulation typically does not end with the award of a licence, and the telco usually benefits from continued monitoring and lobbying of regulatory authorities.

To some readers these may seem like minor questions in comparison to issues of technology choice or financing options, but their answers will form the basis for the differentiation of the company's products from those of its competitors in the minds of prospective customers. While economists often describe telecommunications service as a commodity (e.g., Preist, 1998), to the extent that customers also think this is evidence of a failure of marketing strategy.

To develop effective answers to these many questions requires a detailed understanding of the competitive market environment into which the new service will be launched, and its likely dynamics. Marketers have a number of different frameworks with which to view marketplaces, and some of these are presented in outline form below. Although we present these as discrete concepts, they are usually neither orthogonal nor independent, and multiple iterations of analysis may be necessary before a coherent understanding of the market environment is achieved. In each of these areas, marketers will consider possible competitor actions and reactions.

Market category definition. The market category at issue needs to be defined precisely, and this is often not an easy task for an innovative product or service. For new products, there are no competitors already providing the service (although other companies may also be planning to do so). However, most technology products satisfy some customer need which can be partly or even completely satisfied by some other substitute, technology. The need to make voice calls while away from the home or office, for instance, can be partly satisfied by public telephones, and so these are sometimes seen as partial substitutes for mobile communications services. To the extent that mobile customers make mobile calls while at home or in their offices, then mobile and fixed services may be viewed as competitive. Moreover, if a mobile 
handset is used to play games, the relevant market category may be that of games devices or entertainment and not telecommunications at all.

In addition, many products and services require other, complementary, products or services for effective use by customers. Customers of mobile telephony networks require handsets to be able to use the service, which often leads network operators, usually unwillingly, into the business of handset provision and logistics. These dimensions of substitute and complementary services need to inform the market definition.

Product life cycles. Most marketers believe that all products are subject to life cycles: sales of a new product begin with a small number of customers, grow to a peak at some time, and then decline again, perhaps to zero, as shown in Fig. 1 (Levitt, 1965). Growth occurs because increasing numbers of customers perceive the product as satisfying their needs (which may be diverse). Decline eventually occurs because customers find better means to satisfy these needs, and/or these needs change over time. Most high-technology products are adopted initially only by people with a keen interest in this type of new technology and the disposable income to indulge it. Thus, early adopters are often technologically sophisticated, well informed, wealthy and not averse to any risks potentially associated with the use of a new product. Customers who purchase the product later may do so for very different reasons from those of the early adopters, and may also have different needs being satisfied by its use (Moore, 1991).

For example, in most countries the first adopters of mobile communications services were mobile business and small tradespeople, and wealthy individuals. Only as prices fell have residential consumers and non-mobile office workers become users, and their needs are very different from those earlier in the market. A key

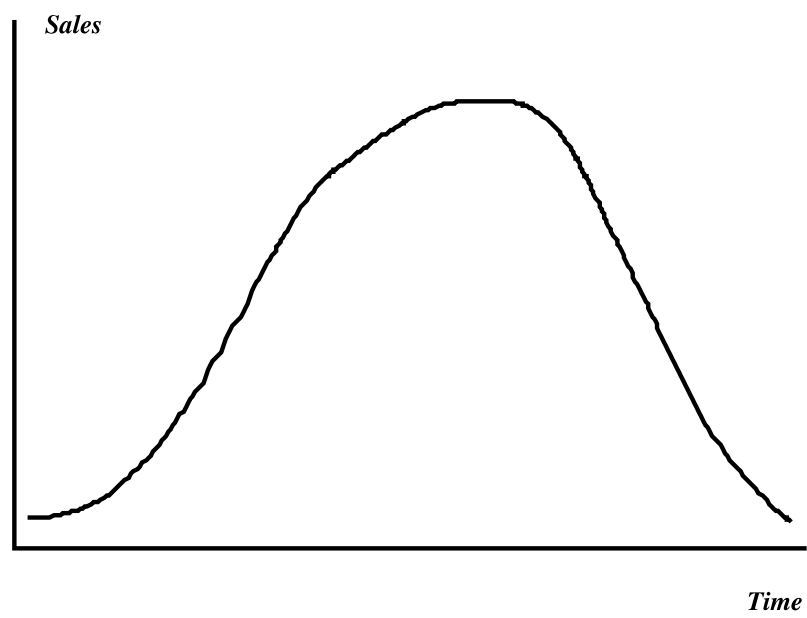

Fig. 1. A product life cycle. 
challenge for the marketing team is to manage the product life cycle, rather than be managed by it. This in turn leads to wide variations in observed product life cycles, for example for the same product in different countries. Fig. 1 shows the generic form of a product life cycle.

Feature bundles. Since Lancaster (Lancaster, 1966; Lancaster, 1971), marketers have viewed products as bundles of features or attributes, which together form the basis of customer preferences for the product. For telecommunications services, the feature set, or bundle, may include basic technical characteristics of the service (such as propagation delays, likely congestion levels, data communications capacities, etc.); value-added or enhanced features (such as voicemail, call diversion capabilities, conference calling, etc.) and commercial elements (e.g., purchase and activation arrangements; pricing structures and levels; billing and payment arrangements; aftersales customer service). Demand will often depend crucially on the particular set of features offered, and different customers may exhibit very different preferences for different bundles (Hemming et al., 1996). In other words, the utility which each customer derives from the product or service is a function of its specific attributes. For example, Vodafone, a British mobile operator, initially launched pre-paid mobile service using a credit-card system where the cards were unusable after a certain date. This was the case even when the card still held a credit balance, a feature disliked greatly by customers.

When customers purchase feature-bundles, they make explicit or implicit tradeoffs between competing features. For example, intending customers of a mobile network may like to have both nationwide coverage and free voicemail. However, one operator may have nationwide coverage but charge for use of voicemail. Another operator may have much lesser geographic coverage but free voicemail. Prospective customers who have a pressing need for wide-area coverage may decide to choose the first operator, even at the expense of having to pay for voicemail. Other prospective customers, with less need for wide coverage, may make the trade-off differently, and choose the second operator.

Segments. Customers may differ in their needs, their attitudes and their behaviours, in where, when and how they wish to purchase and use the product, and in their extent of available income and their responses to price levels and changes. Because of these differences, they may differ in the trade-offs they make between alternative feature-bundles. They may also differ in their willingness to respond to particular advertising and communications media and messages. All these differences can have significant implications for the marketing strategy adopted by the company. Typically, strategies are more effective the more they focus on and respond to differences in customer behaviour of this sort. Such focusing is not without cost, however, and only for the largest corporate customers is it normally cost-effective to develop a marketing strategy targeted to individual customers. For most others, it makes sense to group customers in clusters, called segments, with common needs, attitudes or behaviours (Kotler, 1991; Hemming et al., 1996). Such groupings will be put to different uses within a company, and so different segmentations will be ap- 
propriate at different times and for different uses. A typical segmentation of mobile voice service customers, based on the various needs satisfied by their use of the phone, is presented here. Note that these different segments will most likely have different price sensitivities, will be accessible via different communications media (television, film, magazines, etc.) and may approach purchase decisions in very different ways.

Professional users. Need for communications while in transit to/from office; voicemail and email services; group calling services.

Small business users. Need for communications to support business; voicemail and receptionist services.

Status users. Need for conspicuous consumption; latest technology; stylish design.

Teenage users. Need for connection to social network; voicemail and SMS services and games; stylish design.

Security users. Need for reassurance while driving or in remote locations; little day-to-day need for mobile communications.

Diffusion of innovations. The adoption of an innovation through a community of customers may be considered analogously to the spread of a disease, and so marketers have borrowed mathematical models from epidemiology to model the diffusion of innovations (Bass, 1969; Lilien et al., 1992; Mahajan et al., 1993). These models typically use an S-shaped diffusion curve to model the cumulative sales up to time $t$, for instance:

$$
\text { cum_sales }=\frac{a}{1+b \mathrm{e}^{-k t}}
$$

for constants $a, b, k$. As shown in Fig. 2, this is a curve which grows slowly at the beginning, accelerates quickly through some middle period, and then flattens off to an asymptotic point toward the end of the period of forecasting, as market saturation is reached. Diffusion curves may be thought of as cumulative distribution curves for product life cycles; they have been validated empirically in many markets (Rogers, 1983), and have been applied to telecommunications markets (Altinkemer et al., 1999; Austel, 1990; Frank, 1992). Marketers have good causal explanations for the phenomenon which these curves model, for instance: the differential network, sales and distribution capabilities of the companies serving a target market (e.g. the growth in coverage of new fixed and mobile networks); the differential growth over time in the awareness and education of prospective customers about the products in the market category; the entry of new operators; and the generic product life cycle for the category (Levitt, 1965; Moore, 1991; Urban and Hauser, 1993). However, the selection of the appropriate diffusion model for a new product prior to launch before-sales data exist to calibrate it - is not an easy task, and this is currently an area of academic research in marketing (Roberts, 1998).

When instantiated, these different frameworks for understanding the dynamics of a market may be viewed as the constraints within which a marketing team undertakes 


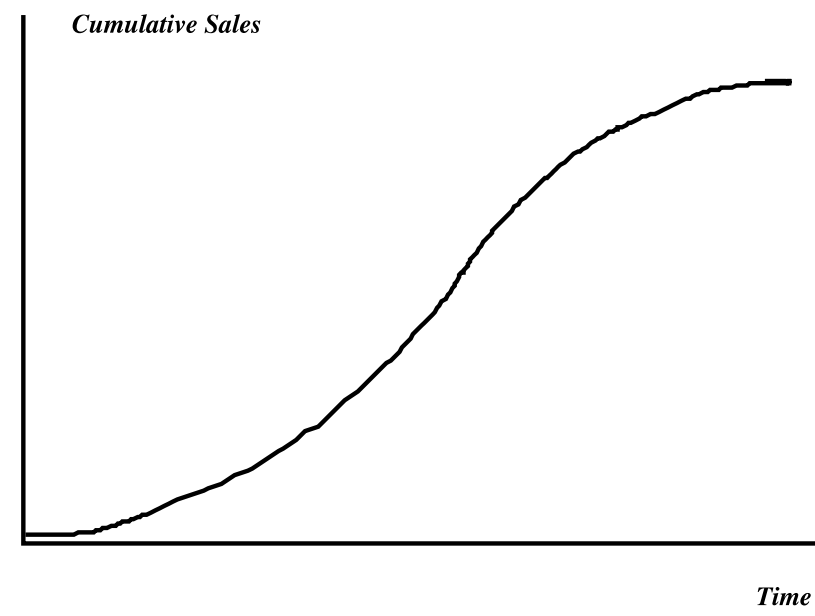

Fig. 2. A diffusion curve.

the development of marketing strategy for a new product or service. As mentioned above, the questions to be answered admit many possible answers, even within such constraints, and marketing strategists almost invariably find themselves involved in making choices between different combinations of answers. Typically, no one combination dominates all the others on every decision criterion, so selection requires trade-offs between different options. For example, do we sell a custom service to a relatively smaller target segment or a "mass market" service to a larger group? If the latter, our gross margins are likely to be less, but so too will be our costs of provision. If the former, our gross margins and our net margins may be greater, but we may require more people with specialized skills (e.g., in service installation) to provide the service; such people may be hard to obtain in a particular country or region. We may also be more vulnerable to a larger competitor entering the market. It is this type of analysis which underpins the demand forecasting activity, and forms the basis for the marketing strategy of the new company.

\section{Why demand forecasts are needed}

Planning a new telecommunications business, as with any new high-technology business, requires a large number of technical and commercial decisions to be made in advance of launch of service. Many of these decisions depend crucially upon knowing the likely numbers of customers and the likely patterns of usage of these customers (what we refer to as "demand"). In the absence of live, operating data, these decisions can only be made on the basis of forecasts of customers and usage.

For a new telecommunications operator, market forecasts are used to guide decisions and actions across all areas of the business, to the point where they become, 
in the words of a former colleague, "the veins of the organization". Since these decisions are wide in scope, and may be unknown to readers without experience of life in a start-up, it is of value to consider some of the uses to which market forecasts are put. Three broad groups of "stakeholders" require demand forecasts: engineering design and implementation teams; marketing and commercial development teams; and external entities, such as potential investors, government regulators, equipment and applications suppliers, and distribution partners.

In the engineering arena, market demand forecasts are needed to guide major technology choices and decisions, and develop detailed technical and service designs. For instance, an intending global mobile satellite services (GMSS) operator has to decide at what orbit to deploy its satellite constellation. The lower the orbit of the satellites, the lesser will be the delay experienced by callers speaking on a satelliteenabled call ("propagation delays") (Brodsky, 1995). On the other hand, the lower the orbit, the faster the satellite will disappear over the horizon (or be obscured by trees or buildings), and so the greater the likelihood of inadvertent call termination (“dropout") (Pattan, 1998; Pope and Hardy, 1999). Different segments of customers may have different preferences regarding these two features, and may trade one feature off against another differently. Thus the selection of a constellation orbit may impact the potential market demand for the service, a fact which led at least one intending GMSS operator to undertake market research to assess the potential customer tolerance of propagation delays before selecting an orbit (Wetenhall, 1998).

In addition to design questions, forecasts are needed by the engineering implementation team to configure and dimension the network and its elements. In a mobile network, for example, calls typically need to be routed along fixed links between base stations and mobile switching centres, and between switching centres. Whether such links are to be leased from fixed network operators or constructed, good prior indications of traffic along them will be needed to make the necessary financial and contractual commitments. Such commitments may be required a year or more before there are any operational traffic data on which to base a forecast. Similarly, contractual commitments to equipment vendors are almost always dependent on estimates of demand, and must be made well in advance of the launch of service.

In the marketing and commercial arena, demand forecasts are required to quantify potential customer segments and geographic territories for prioritization by the business. In the GMSS market, for example, Iridium initially said that frequent International Business Travelers were a key target segment (Hardy, 1997), while its competitor ICO Global announced four distinct target segments, defined around applications (ICO, 1999). Such prioritization guides decisions across the entire company, from design of service features to selection of distribution partners. Likewise, the prioritization of geographic focus is often a crucial element of business planning, and effective prioritization requires estimates of demand, usage, revenues and costs by region. The launch in 1993-94 of personal communications services 
(PCS) networks in Britain, for instance, saw the two new operators adopt radically different geographic launch strategies: Mercury One2One launched service only in and around London (inside the perimeter of the M25 Motorway), while Orange, launching several months later, offered its service nationwide. By focusing on a smaller launch area, One2One was able to beat its competitor to market. However, Orange was able to offer its customers nationwide coverage from the outset (which One2One did not match for several years), and, for this and other reasons, soon had the greater market share of the two. ${ }^{1}$

Moreover, as with configuration of engineering elements, demand forecasts are needed to configure and dimension the commercial elements of the business. Commercial questions which must be answered prior to launch of service and which depend on the forecasts include: Where should retail sales outlets be located? How many of them should there be? How large should each be? How large a target customer area should each serve? What sales throughput should be expected in each? What performance targets should be established for each? etc. While these issues may appear small relative to some of the technical issues - such as the selection of satellite orbits, for example - without an effective retail presence sales will not achieve targeted levels and the company's financial position will suffer. In addition, the complexity of deciding these commercial issues and possibly implementing them across multiple countries and cultures simultaneously means many decisions must be made considerably ahead of commercial launch of service. Identifying and shortlisting potential downstream distribution partners in foreign countries, and negotiating commercial agreements with them, may take as long as two years, for example.

In addition, demand forecasts are needed to calibrate models of the company's revenues, profits and cash flows, to assess business viability, to determine cash, equity and borrowing requirements, and to determine appropriate pricing structures and levels. For start-up companies, financing may be very uncertain and so much may depend upon the forecasts and financial models generated.

Such financial models are also used in the third arena of application of the demand forecasts, that of convincing external parties to support the venture. Financial models are needed to convince potential investors, lenders, stock exchange regulators and other business partners of the market potential and of the company's commercial viability. This is especially important of products creating a new category, where there may be considerable - and justifiable - doubt outside the company and its investors about the market potential for the product. Likewise, third-party vendors, such as manufacturers of user terminal equipment and providers of applications software, must often be persuaded that a commercially viable market exists for

\footnotetext{
${ }^{1}$ Note that, in some countries, such as the USA, a distinction is made between so-called cellular operators, who were generally the first companies given public mobile licences using call hand-off technologies, and PCS operators who were given licences later. Cellular and PCS companies may or may not have different technologies, marketing strategies or target customers.
} 
their products and services. Because vendors are not always so persuaded, new telecommunications service providers are sometimes forced to underwrite their vendors' investment risks; British mobile operators, Mercury One2One and Orange, as the world's first PCS operators, had to do this for their handset vendors in the early 1990s (Hadden, 1995). Likewise, the GMSS operators, Iridium, Globalstar and ICO, had to adopt similar approaches with their user terminal vendors and logistics managers to ensure global terminal availability at launch.

In the case of new telecommunications services, operating permits are usually necessary before service can be provided, and regulatory agencies often need to be persuaded that a sufficiently large potential market exists before they will award the necessary licences for new services. For example, new wireless services may require frequency allocations from national and global regulatory authorities, especially where existing users occupy the relevant frequency bands. Specialized services may also require additional licensing arrangements, such as the aviation authority approvals required in most countries before new communications equipment can be fitted to aircraft. As mentioned earlier, telecommunications regulators in many countries also require that prices be filed publicly prior to their taking effect, so that prices may need to be finalized well before commercial launch of service.

In summary, there are many stakeholders with a legitimate and abiding interest in having forecasts of demand and usage, both within and outside a company planning to offer new telecommunications services. The interests of these stakeholders may be widely divergent, and most will have their own perspectives of the marketplace and its dynamics, and their own assessments of market demand. They may also have widely different levels of appreciation of the technical difficulties inherent in any forecasting activity. Such is the environment in which the market planning team will typically be required to produce market forecasts.

\section{Forecasting methods}

Having briefly discussed the need for market demand forecasts, this section presents a high-level introduction to the main methods currently used by marketers to generate these forecasts for new communications products.

\subsection{Exploratory methods}

There are a number of methods marketers use to generate demand forecasts which do not involve collecting of primary data from prospective customers. These methods are sometimes referred to as exploratory methods, and sometimes as qualitative methods, although the latter term also includes various non-statistical methods of customer data collection (such as Focus Groups).

One approach used to structure thinking about the future in a turbulent environment is scenario analysis (Schwartz, 1991). This method has participants consider 
possible alternative futures and then explore the consequences of these futures. Typically, the participants are drawn from the ranks of senior management of the company, although outsiders may also be included. Having articulated possible futures, participants may also then seek to identify causal influences on these futures, and the relationships between influences. For example, increases in consumer leisure time may lead to increased use of communications devices for games and entertainment pursuits; demand for a specific telecommunications application may therefore ultimately depend upon the extent of disposable time which customers have. Further, one can also assign subjective measures of uncertainty to the influences (Brauers and Weber, 1988). The resulting influence diagram can be used to produce a probability distribution on the variable(s) of interest, from which expected values may be derived as forecasts. This approach is termed structured scenario analysis, and has been used to forecast demand for new telecommunications services (Gruszecki and Andries, 1990), as well as for other products in markets undergoing turbulent change (Robinson, 1984). ${ }^{2}$

However, such an approach requires reaching an agreement on the structure of influences and an assignment of uncertainty values to influences. For new market categories in turbulent environments, there are typically a large number of potential causal influences, and the relationships between them may well be very complex. In these circumstance, seeking agreement on subjective inputs to complex models, is usually very difficult. A related approach is to assign subjective degrees of belief to the scenarios themselves (McBurney and Parsons, 2001), and update these belief assignments as new information arises or as subjective beliefs change. This method, applying a belief function model, uses non-probability representations for uncertainty first developed by the artificial intelligence community (Parsons and Hunter, 1998), and increasingly applied in domains where knowledge is limited. The use of belief functions can accommodate, in a coherent fashion, the different subjective views about the future which may exist within an organization at such times.

Scenario analysis methods seek to articulate the range of possible alternative futures, and thus they tend to expose and highlight differences in views about the future. Another approach, called the Delphi method, generally seeks to achieve a consensus rather than expose differences. In this method (Jolson and Rossow, 1971), a group of experts are questioned individually (and often anonymously) about their opinion of some future event, and their reasons for their opinions. The results are then aggregated, and circulated back to the group. The participants are then again asked for their opinions, which may have changed in the light of the information

\footnotetext{
${ }^{2}$ Note that the application of probabilities to scenarios is a contentious issue in the business planning community. See, for example, the debate conducted on the Global Business Network's web-site (www.gbn.com) in the early 1990s, and later published there under the title "Probabilities: help or hindrance in scenario planning?"
} 
received about their peers' opinions, and the process repeated. Eventually, either a consensus emerges or the reasons for its non-emergence generally become evident. This is a common research technique for situations involving great future uncertainty, and participants are usually selected on the basis of their public status as experts, for example, regulators, academic commentators, journalists, etc. The Delphi method is less commonly used for participants from within an organization because differences in opinion within one company can be exposed more readily through direct conversation, and a consistent viewpoint imposed upon the participants by management decision.

\subsection{Primary market research techniques}

When compared with alternatives, exploratory techniques are generally inexpensive and fast to implement. They tend to generate results based on subjective views of the marketplace, and so are not always very forceful in persuading others. More persuasive, in general, are forecasts based on primary market research, which are those involving the collection of data through interviews with prospective customers (Aaker et al., 1995; Green et al., 1988). However, as we discuss in Section 5, forecasts using primary market research may be no more reliable than forecasts using exploratory methods.

Primary data are usually collected through some form of sample survey, in which interviews are conducted with prospective customers or purchase-decision-makers (who may not be the same people as the users of the service). Survey interviews may be conducted by e-mail or letter, by telephone, or face-to-face. Typically, costs per interview increase in this order, as does the effective response rate. (Response rates to postal surveys may be as low as $1 \%$ or $2 \%$.)

A survey will typically describe the proposed product or service in some form, and then ask the respondent about their likely purchase intentions and anticipated usage. Questions will also be included on the socio-demographic characteristics of the respondent (e.g., their age, income, marital status, etc.), in the case of individuals, or the corporate equivalents ("firmographics"), in the case of an organizational respondent. Questions on lifestyles, attitudes and leisure activities are also common. Responses to these background questions are matched against responses to the intentions questions, so as to enable understanding of the characteristics of customers who are attracted to the proposed offering, and how best to communicate with, and sell to, them.

For new services which may be complex bundles of features, a key marketing task is to decide what features to be included in the service package. Customers will purchase such bundles only after making a trade-off between the different feature-bundles available to them at the time of purchase. A market research technique known as conjoint analysis is often used to simulate and model such multi-attribute purchase decisions (Green and Krieger, 1993; Urban and Hauser, 1993). Purchase intention models in marketing can be extremely complex. Even 
purchase advice that one prospective customer gives to others - what marketers refer to as "word-of-mouth" - can be simulated and its impact calibrated, as in Urban et al. (1990, 1996).

Conjoint studies generally ask customers to rank hypothetical products in order of preference, sometimes when these products differ by just one feature or one level of one feature (e.g. one price level versus another price level, price being considered as one feature). In this way, we can calibrate the extent to which one feature is preferred over another. In particular, if price is included as one of the features, an estimate of the price sensitivity can be obtained, and an understanding of how price is traded against other features. For example, a new mobile operator may have lesser geographic coverage at launch than do its incumbent competitors, and so will typically offer services at lower prices. The challenge facing the marketing team is to make the price differential large enough to attract customers who are willing to trade coverage against price, but not larger than necessary, and certainly not so large as to attract more customers than the network can support. Conjoint studies can enable such fine calibration to be achieved.

Armed with the results of a conjoint study, marketers can then estimate the expected demand for each combination of product features (each bundle) based on the percentage of customers who preferred this bundle over the offered alternatives. However, asking respondents in an interview to make comparisons of feature bundles is time-consuming, and there is a limit to how many comparisons an individual respondent can make. In addition, conjoint surveys are generally most effective when administered face-to-face. These considerations result in conjoint analysis being an expensive form of primary market research.

\subsection{Market models}

The approaches described above are essentially methods for collection of market data - either from experts or from prospective customers - which can be used to develop a market forecast. How are the forecasts actually generated from this information? There are a number of ways in common use in telcos, which we collectively group under the heading market models. We may think of a market model as simple input-output device, where the inputs are some assumptions about our product offerings and their expected marketplace environment, and the model output is a forecast of the market demand for the product. The input assumptions may be quantitative or qualitative, as indeed may be the outputs. The form of the model that is, the function which transforms inputs to outputs - may be simple or complex. We now discuss at a high level a number of models in common use in telcos for new product forecasting.

Subjective methods simply involve asking participants (e.g. Delphi participants or scenario planners) to state their opinion about the likely market size. These may be point-estimates or interval estimates, and may be combined with measures of uncertainty (e.g. "There is a 30\% chance of demand being greater than 2 million, and a 
$70 \%$ chance it will be less than 2 million.") Opinions from different people may be combined or averaged in an appropriate manner, as in McBurney and Parsons (2001) and UMTS Forum (1999). A refinement of simple subjective approaches is to use some form of hierarchical model. Thus, for example, an intending mobile satellite operator may target only existing users of terrestrial mobile services, and so customer forecasts for satellite mobile services may therefore be expressed as a percentage of the customer numbers of land mobile networks. Again, expert opinion can be sought on the appropriate level of this percentage and the expected total demand for terrestrial services.

Comparative approaches forecast demand using the experience of a cognate market or country. We might, for instance, forecast the demand and uptake of cable television (CATV) services in a country where this is a new technology either by using similar CATV figures from other countries or by using demand and diffusion figures for broadcast television in the same country. Because new services usually seek to satisfy different needs to existing services, this approach does not always generate reliable forecasts. Second generation cordless telephone (CT2) services, for instance, were expected by many people to repeat the earlier successes of analogue cellular services, as the technologies are very close. However, they failed to do so, at least in Western countries, arguably because, with lesser functionality than cellular, they did not meet the expectations held by consumers who knew about cellular.

Primary research surveys of purchase intentions are used to generate demand forecasts in a number of ways. If we simply ask respondents "Do you intend to purchase this service?", then we can estimate the total demand for the service by multiplying the percent who answer positively by the total population under consideration. (Such an inference is only valid statistically, of course, if the sample of respondents is representative of this population.) If we had asked a more sophisticated question of purchase intentions, such as: "How likely is it that you will purchase this service in the next year? Very likely, likely", etc., then we can produce a finer analysis. We might simply conclude, say, that $20 \%$ of the population is very likely to purchase the service in the next year. Or, we might add together the numbers of those responding "very likely" with those responding "likely" to give a better estimate of demand. In fact, standard market research practice is to take a weighted average of all those responses which were not negative, for example, $80 \%$ of those responding very likely plus $65 \%$ of those responding likely plus $50 \%$ of those responding "neither likely nor unlikely. Such weightings are justified on the basis that not all respondents will later do as they indicated, but (it is argued), the more extreme the intention, the more probable it is that the intention will be effected. If information has been collected on socio-demographic and similar variables, these may be used to produce demand forecasts for separate segments, such as for large, medium and small businesses. If use has been made of stratified random sampling (a statistical technique in which separate sub-samples are taken from each segment of the population, Chaudhuri and Stenger, 1992), then segment-level forecasts may be 
aggregated to produce a total population forecast based upon the relative sizes of each segment in the total population.

Conjoint analyses may be used to generate demand forecasts in the same manner as for basic surveys of purchase intentions, except that the forecasts may be produced separately for each bundle of features included in the conjoint survey. In this way, we may assess the potential market demand for different product definitions. If we have knowledge on the possible feature bundles being considered by competitors, we may also include those in the conjoint model, and so generate likely market shares for each intending provider in the same way.

This description of the demand forecasting calibration methods should indicate that forecasting processes in common use are straightforward, at least at a conceptual level. However, this is not to say that the resulting forecasting models are not large. They may be very complex, with long chains of causal or hierarchical reasoning (McBurney and Parsons, 2001), and with many, diverse ratios and weighting factors to be estimated. The largest demand forecasting model in our experience was $6.6 \mathrm{~Gb}$ in size, with the file of input assumptions alone exceeding 55 $\mathrm{Mb}$. Estimating the satellite capacities needed for an intending global satellite network, for instance, requires estimates of the usage likely to be generated at each point (or small region) on the earth by users calling to each other point, by each time of day. If the network in question permits mobility of terminals, then we may need to disaggregate this forecast by the country of registration of the calling party, for example, to respond to specific national requirements for call routing or call interception, or to reliably estimate revenues for those services priced nationally. The resulting traffic forecasting models are both large and conceptually complex.

\subsection{Post-launch forecasting}

Forecasting demand does not end with the launch of commercial service. Indeed, the launch of new products and services should not, and usually does not, end with the initial launch, and new product development and deployment plays an increasingly important role in network evolution and business growth for most telcos. Each new product in a product portfolio will require demand forecasts to be prepared, not least because, in a well-managed company, a separate market-entry decision (GO versus NO GO) should be made for each.

Moreover, total market demand - the potential or saturation level of the market still needs to be forecast, even after a product is commercially available. Because of the problems associated with collecting primary market research data for a new product category, one of us has previously argued (Hemming et al., 1996) that some of the attention paid to pre-launch forecasts is misplaced, and a key focus of telcos should be the creation of systems and processes to enable rapid learning from and adjustment to the marketplace after launch of service. These comprise an entirely different set of forecasting issues which are not discussed here. 


\section{The challenges of forecasting demand}

Having briefly outlined the main techniques used by marketers to forecast communications demand, we now discuss some of the key problems involved in their use.

\subsection{Conceptual issues}

Forecasting of demand for a new service requires an understanding of the marketlevel dynamics of the relevant marketplace, and this understanding is not always easily obtained. Traditional methods of forecasting demand - such as time series analysis and econometric modelling, e.g., Kridel and Dolk (1993) and Lee (1988) require historical data on the market concerned or on a closely related one. Such data is not available for innovative products which, in the language of marketing, define a new market category (Kotler, 1991). Even when data from analogous markets are available, a turbulent market environment may render past data less than useful, as it may inhibit the forecaster from identifying new opportunities, changes in trends, market discontinuities, etc. (Gruszecki and Andries, 1990). Traditional methods of forecasting have been likened to "looking forward through a rear-view mirror" (Koreisha and Stobaugh, 1979). In one typology of business environments in terms of their levels of uncertainty (Courtney et al., 1997), the environment faced by companies proposing a new telecommunications service would be either Level 3 ("A range of futures") or Level 4 ("True ambiguity"), on a four-point scale.

In addition to the major dynamics of a marketplace, demand for a new product or service may be very sensitive to the specific product features it comprises. These features will depend upon technical, regulatory and financial constraints and design decisions made by each intending operator, and so are likely to differ from one operator to another. Different feature-bundles are likely to appeal differentially to different customer segments, and thus have an impact on the market demand for each company's offering. At the early stages of business planning for a new product, considerable latitude exists for each company regarding the product and service attributes, and consequently considerable uncertainty exists as to the likely levels of possible demand. In addition, the long planning and implementation periods required for many new telecommunications services may add to the forecasting uncertainty. For example, in the case of GMSS, where lead times between project inception and commercial launch have typically been eight or more years (Anon, 1999), customer expectations and preferences will almost certainly have changed considerably through the planning period, not least because other product offerings complementary and substitute - have not remained constant in this time.

Thus, conceptual challenges to demand forecasting arise from both market-level and product-level factors. The terrestrial mobile communications industry provides a good example of the difficulty of forecasting demand for a new category in a turbulent environment. Public mobile communications services based on cellular 
technologies were introduced from the early 1980s, starting in Scandinavia and Japan. Services were then launched in North America and elsewhere in Western Europe from the middle of the 1980s and have since been introduced in most countries around the world. The services were completely new to customers, to operators and their distribution channel partners, to equipment vendors and suppliers, and to government regulators. In addition, the last 15 years have been a period of great change in the telecommunications industry, due to technological innovations, privatization and corporatization of state-owned enterprises, deregulation and opening of telecommunications markets leading to the entry of new competitors to the market, and changing customer preferences. As a consequence, forecasting of demand has been a difficult task. The head of forecasting and analysis for the International Telecommunications Union has stated "the mobile communications market has proved to be one of the most dynamic, but also one of the most difficult to forecast, of all the parts of the telecommunications sector. The forecasts produced by equipment suppliers, network operators and by specialist consultancies have consistently underestimated the actual market potential" (Kelly, 1996). Even by 1995, when forecasters had a decade's worth of experience of the rapid growth of demand and the category was no longer new to customers or to operators, forecasts from credible forecasters for worldwide terrestrial mobile customers in the year 2000 - just five years ahead - ranged from 200 million to 350 million customers (Kelly, 1996). Yet even the largest of these may have been underestimates. In early 1999, a mobile industry technology association forecast the worldwide year 2000 demand at 426 million customers (UMTS Forum, 1999).

This example raises the deeper conceptual issue of what counts as a good forecast. All forecasts for new products are inaccurate, and predictive accuracy is not a good performance measure when those developing the forecast have potential to influence the direction of the market. An alternative approach is to consider the question not as one of accuracy but as one of persuasiveness: How much do the reasons proposed for a particular forecast compel belief in it? Does it withstand scrutiny, at least to the level required by the users of the forecasts? Are the underlying assumptions justified and consistent? In asking these questions, one would be challenging the marketing understanding behind the forecast, i.e., the scope of issues presented in Section 2. In responding to such questions, the forecasters will need to have a good understanding of the likely shape of future market demand before undertaking a detailed estimation of it: Will demand grow quickly or slowly, for example, or will its growth depend on that for some other product? Will demand depend on the number of competitors in the market? etc. The evidence presented in answer to such questions may be subjective or based on primary market research data. As we discuss below, the latter data are not without their own difficulties of validity and reliability when used for forecasting demand for new products. Because we believe the focus needs to be on arguments for the forecast, we support a statement by Walsham (1982), developing 30 -year demand forecasts for telecommunications services, that a model needs to be regarded as "a forum for debate rather than a forecasting tool". 


\subsection{Data collection issues}

The large financial stakes often at risk by companies in offering new telecommunications services make the case for undertaking primary research as the basis for demand forecasts compelling. For example, some $\$ 50$ billion had been requested of investors before launch in global satellite services (Evans et al., 1998) and much of this amount was invested or pledged. In most cases, investors only did so after seeing the results of primary market research which suggested that demand for GMSS would be sufficiently large. Yet the recent experience of the industry, with two of the three operators, Iridium and ICO, descending in bankruptcy, would suggest otherwise. While there are many reasons for the ultimate failures of the GMSS operators, their ventures would not have proceeded as far as they had without primary research indicating a sufficiently large market demand. Primary market research data, however, are not necessarily reliable, for a number of reasons.

Firstly, as mentioned above, demand may depend crucially on specific service and quality features, and these are not known at the outset of the planning activity, when forecasts of demand are first needed. Indeed, one purpose of primary market research is to gather information relevant to their specification (Urban and Hauser, 1993). In any case, telecommunications features are difficult and costly (although not impossible) to simulate in a market research interview. Likewise, actual demand will also depend on what alternatives, partial or complete substitutes, and complementary services are available to customers at the time of their purchase decision.

Secondly, even if interview respondents were to know and appreciate the exact features of a new service and of competing services, respondents are not necessarily accurate predictors of their own future behaviour. As mentioned earlier, market research surveys of purchase intentions typically ask prospective customers to rate their likelihood of purchase using terms from a qualitative linguistic scale such as very likely, likely, unsure, unlikely, and very unlikely (Kotler, 1991). Even though such a scale permits some uncertainty about future intentions to be represented, respondents may act differently when placed in an actual purchase situation. One report, talking of the use of linguistic labels with crisp-set-based conjoint models to forecast market share in existing market categories, noted that: "The resulting models are inflexible, costly to implement and have predictive validity not substantially better than chance" (Turksen and Willson, 1995). This is likely to be even more the case with a new product category, such as mobile satellite services, where early market research surveys have necessarily been conducted several years in advance of the service launch. Moreover, individual respondents typically find it difficult to factor network effects into predictions of their own behaviour: the actual individual benefits to be gained from using some new product, such as access to the Internet, may depend crucially on how many other people have access to it, yet no one respondent is likely to know this.

Finally, primary research is often unreliable for new telecommunications services because many of these services are transnational or global in nature, and so demand 
estimates require market research to be conducted across the globe. Different cultures, however, accord different statuses and meanings to a market research interview and to the questions within it (Stepick and Stepick, 1990), and statistical market research techniques do not always transfer readily across cultures (see, for example, Casley and Lury, 1981; Kushner, 1982; McBurney, 1988; Stanton et al., 1982). Since even simple concepts such as "spouse" (Stepick and Stepick, 1990) and "father" (McBurney, 1988) are understood very differently in different cultures, how much more so for abstractions such as "purchase likelihood" with regard to not-yet-existent services. ${ }^{3}$ For some customer segments, such as Multi-National Corporations or International Business Travelers, for example, there may be enough commonality between respondents in different cultures for this not to be a problem. For other segments, such as villagers in remote areas of developing countries, cultural issues associated with doing market research itself are likely to be a major source of unreliability.

Another challenge for primary market research which often arises is the fact of sparse target populations. Although one estimate for the global market for GMSS, for example, was thirty million or more customers by 2005 (Rhinds, 1999), the total number of terrestrial mobile customers worldwide at this time may exceed 1 billion (Ovum Ltd., 1999). Indeed, one mobile industry group recently predicted 1.7 billion terrestrial mobile customers globally by 2010 (UMTS Forum, 1999). Hence, GMSS demand will be a small percentage of the total terrestrial mobile demand. If potential GMSS customers comprise, say, $2 \%$ of existing mobile users then we would need to interview an average of 50 terrestrial mobile users to locate just one potential GMSS customer. In order to make statistically valid comparisons between potential GMSS customers (for instance, according to their country of origin or their frequency of usage) we may require a realized sample size of several hundred GMSS respondents (Aaker et al., 1995; Green et al., 1988). To achieve 300 potential GMSS users would thus require interviews with 15,000 cellular customers, a sample size that would be prohibitively expensive.

In such circumstances, it is common for market researchers to use a simple screening question or questions (e.g., "Are you an existing cellular user? If so, do you ever travel outside cellular coverage areas?") in, say, a telephone survey, and then arrange to follow this with a more detailed face-to-face interview for those passing the screen (the targeted group). That is, only those who have answered "Yes" to the initial screening questions are administered the full questionnaire. Such an approach would provide the detailed information required on the attitudes, preferences and behaviours of the target customers but possibly at the cost of lesser information as to their prevalence within the wider population, and their relative characteristics.

\footnotetext{
${ }^{3}$ Indeed, some anthropologists (e.g., Raven, 1996) even argue that not all cultures use the same rules of inference in logical reasoning.
} 


\subsection{Organizational issues}

A further challenge for demand forecasting of new telecommunications services arises from organizational issues. The first of these is what may be termed opinion proliferation. The combination of an absence of comparable historical data and the unreliability of primary market research means there is generally no one single prevailing viewpoint regarding demand levels within an intending service provider and among its stakeholders. Everyone has an opinion on the size and dynamics of the market, and there is often no agreed or objective way to arbitrate between these. Primary market research findings might conceivably provide a resolution, were they not subject to so many challenges of reliability and validity. A related problem arises from the diverse nature of stakeholder requirements for forecasts, which often leads to a tension between the feasibility of the forecasting activity and the granularity of the resulting forecasts. Survey respondents have finite time and patience, for example, and adding further questions to a survey can make it unworkable. Such tensions are not always apparent to users of forecasts.

As we have shown, planning of the business cannot proceed without some forecasts of demand, however tentative. Moreover, many of the business planning questions involved are complex and inter-related (Hardy, 1996), and can only be resolved knowing the results of the others. Which satellite orbits a new GMSS operator will select, for example, will depend partly on the expected levels of demand, which will, in turn, depend on the expected pricing strategy, which will be dependent on the amount of free cash available to the company at launch, itself a function of investment and capital expenditure levels, which may again depend on the satellite orbits selected. Because the relationships between such variables are unknown and are almost certainly non-linear, resolution of these questions simultaneously is generally impossible. Consequently, interim solutions are typically adopted, with periodic revisions through a process of iteration across the company. Some decisions, such as the choice of orbit in a satellite business (which choice influences the nature of satellites to be manufactured), need to be made early in the design process and can only be revised with prohibitive financial costs and delays. Thus, such decisions tend not to be revised very often. Other decisions, however, such as the type of retail outlets to be used, may be postponed until much closer to the launch date of commercial service and may even be revised subsequent to launch, if necessary.

In this situation, any useful demand forecasting activity must be iterative, with successive forecasts being adopted by the company as its current official forecasts. This decision process is very similar to the so-called "blackboard architecture" for complex decision-making used in intelligent computer systems (Nii, 1986; Parsons et al., 1993). Despite this similarity, it is not common for start-up telecommunications companies to formalize this process with a blackboard-like decision process. We believe there is considerable value in doing so. 


\section{Conclusion}

We have written this paper primarily to inform non-marketers about the diverse reasons forecasts are developed by a new communications company, and the methods commonly used by marketers when developing forecasts. Lack of knowledge of the context and methods of forecasting by those not involved in the process often contributes, in our experience, to a gulf between marketing and other departments in a company. Also common is a belief that forecasts are simply invented. None of us knows the future, so no forecast can ever be entirely accurate. The relevant question as we have argued, is not predictive accuracy, but persuasiveness: are we convinced by the arguments put forward in support of a particular forecast.

Our forecasting experience has led us to several beliefs about effective management of the forecasting process. Firstly, that simple forecasting models are better than complex ones, because of the need for forecasts to be persuasive. If users of the forecasts do not understand their derivation, then they are less likely to be persuaded by them. Moreover, for many new services, the competitive and financial pressure to launch as quickly as possible mitigate against large and time-consuming primary data gathering projects. In these circumstances, subjective or comparative forecast models may be all that can be done in the time permitted. If they are convincing to the stakeholders, than they will be fit-for-purpose, even though simple.

Secondly, forecasts need to stay aligned with the company's marketing, technical and financial strategies. This is not easy to achieve in the planning phase of a startup, when these strategies are still being created. The need for ongoing alignment leads us to recommend a formal, blackboard-like, process for managing forecast development and dissemination within a start-up, as mentioned earlier. Such a formal process also helps instil a culture of reflective iteration within the company, by which we mean that everyone realizes that the forecasts (and indeed other plans) are only always tentative, and may be revised in the light of new information received and validated. While corporate reflection may be seen as a delaying tactic, we believe that embedding it in a formal process of iteration can act to increase the speed and agility of the company in responding to changes in its external environment.

It is common, in our experience, for forecasts, once produced, to take on a life of their own. Once published, especially after external publication, they appear to many involved to be sacrosanct. This is unfortunate. Our third belief is that the process of forecasting, and the quality of forecasts produced, would benefit from wider understanding of the challenges involved, and the limitations of the techniques available. We have sought by means of this article to address this issue.

\section{Acknowledgements}

Work on this paper commenced while the first two authors were members of the Department of Electronic Engineering at Queen Mary and Westfield College, Uni- 
versity of London, and was partly funded by the British Engineering and Physical Sciences research Council (EPSRC), under grant GR/L84117 and a studentship. We are grateful to Martin Garner of Ovum for his detailed comments on an earlier draft. The views expressed here are those of the authors only, and are not necessarily those of our current or former employers or consultancy clients.

\section{References}

Aaker, D.A., Kumar, V., Day, G.S., 1995. Marketing Research. Wiley, New York.

Altinkemer, K., Yue, W.T., Yu, L., 1999. Adoption of low earth orbit satellite system: a diffusion model under competition. In: Proceedings of the Seventh International Conference on Telecommunications Systems Modeling and Analysis. American Telecommunications Systems Management Association, Nashville, TN, USA, pp. 339-355.

Anon. Iridium reaches debt waiver as it struggles to win customers. Wall St. J., 1 June 1999.

Austel. Public mobile telecommunications services. Report to the minister of transport and communications, Australian Telecommunications Authority, Commonwealth of Australia, Melbourne, Australia, 1990.

Bass, F.M., 1969. A new product growth model for consumer durables. Manage. Sci. 15, $215-227$.

Brauers, J., Weber, M., 1988. A new method of scenario analysis for strategic planning. J. Forecasting 7 , $31-47$.

Brodsky, I., 1995. Wireless: The Revolution in Personal Telecommunications. Artech House Publishers, Boston, MA.

Casley, D., Lury, D.A., 1981. Data Collection in Developing Countries. Oxford University Press, Oxford, UK.

Chaudhuri, A., Stenger, H., 1992. Survey Sampling: Theory and Methods. Dekker, New York.

Courtney, H., Kirkland, J., Viguerie, P., 1997. Strategy under uncertainty. Harv. Bus. Rev. 75 (6), 6679.

Evans, A.L., Rose, J.S., Venkataraman, R., 1998. The future of satellite communicating. McKinsey Quart. 2, 7-17.

Frank, B., 1992. Internationale entwicklungsmuster zellularen mobilfunks. In: Kruse, J., Schenk, G. (Ed.), Zellularer mobilfunk: Neue märktemit neuen netzen für das funktelefon, R. v. Decker's Verlag, Heidelberg, Germany, pp. 271-281.

Green, P.E., Krieger, A.M., 1993. Conjoint analysis with product-positioning applications. In: Eliashberg, J., Lilien, G.L. (Eds.), Handbooks in Operations Research and Management Science, vol. 5. Marketing, North-Holland, Amsterdam, The Netherlands, pp. 467-516.

Green, P.E., Tull, D.S., Albaum, G., 1988. Research for Marketing Decisions, fifth ed. Prentice-Hall, Englewood Cliffs, NJ.

Gruszecki, M., Andries, R.N., 1990. Some new concepts in demand and traffic forecasting and planning of future telecommunications services. Comput. Networks ISDN Syst. 20 (1-5), 65-74.

Hadden, A.D., 1995. Personal Communications Networks: Practical Implementation. Artech House Publishers, Boston, MA.

Hardy, Q., 1996. How a wife's question led Motorola to chase global cell-phone plan. Wall St. J., 16 December 1996.

Hardy, Q., 1997. Iridium creates new plan for global cellular service. Wall St. J., 18 August.

Hemming, P., Shuker, D., McBurney, P., 1996. Innovative PCS Marketing: How to Build and Execute a Winning Marketing Plan. Redwing Consulting Ltd., Dallas, TX.

ICO, 1999. ICO Facts. ICO Global Communications Ltd., Hammersmith, London, UK, March.

Jolson, M.A., Rossow, G.L., 1971. The Delphi approach in marketing decision making. J. Marketing Res. $8,443-448$. 
Kelly, T. 1996. Forecasting the Mobile Communications Market: A Finger in the Airwaves? Paper presented to Conference on Market Forecasting in the Telecoms Industry, Hong Kong, March 1996, International Telecommunications Union, Geneva, Switzerland.

Koreisha, S., Stobaugh, R., 1979. Limits to models. In: Stobaugh, R., Yergin, D. (Eds.), Energy Future: Report of the Energy Project of the Harvard Business School, Random House, New York, pp. 234 265.

Kotler, P., 1991. Marketing Management: Analysis Planning Implementation and Control. Prentice-Hall, Englewood Cliffs, NJ.

Kridel, D.J., Dolk, D.R., 1993. Modeling telecommunications demand analysis. Interfaces 23 (2), 3-13.

Kushner, J.M., 1982. Market research in a non-Western context: The Asian example. J. Market Res. Soc. $24,116-122$.

Lancaster, K.J., 1966. A new approach to consumer theory. J. Political Econ. 74, 132-157.

Lancaster, K.J., 1971. Consumer Demand: A New Approach. Columbia University Press, New York.

Lee, J.C., 1988. Nested Rotterdam model: applications to marketing research with special reference to telecommunications demand. Int. J. Forecasting 4, 193-206.

Levitt, T., 1965. Exploit the product life cycle. Harv. Bus. Rev. 43 (6), 81-94.

Lilien, G.L., Kotler, P., Moorthy, K.S., 1992. Marketing Models. Prentice-Hall, Englewood Cliffs, NJ.

Mahajan, V., Muller, E., Bass, F.M., 1993. New-product diffusion models. In: Eliashberg, J., Lilien, G.L. (Eds.), Handbooks in Operations Research and Management Science, vol. 5. Marketing, NorthHolland, Amsterdam, pp. 349-408.

McBurney, P., 1988. On transferring statistical techniques across cultures: The Kish Grid. Curr. Anthropol. 29 (2), 323-325.

McBurney, P, Parsons, S. 2001. Using belief functions to forecast demand for mobile satellite services. In: Mock, T., Srivastava, R.P. (Eds.), Belief Functions for Business Decisions, Physica-Verlag, New York, in press.

Moore, G.A., 1991. Crossing the Chasm: Marketing and Selling High-Tech Products to Mainstream Consumers. Harper Collins, New York.

Nii, H.P., 1986. Blackboard systems (part one): the blackboard model of problem solving and the evolution of blackboard architectures. The AI Magazine, Summer, pp. 38-53.

Ovum Ltd. Cellular Connection Forecasts. Report of the Mobile and Satellite Communications Group, Ovum Ltd., October 1999.

Parsons, S., Brown, T., King, S., Mamdani, E.H., 1993. A blackboard system for active decision support in configuring telecommunications services. In: Proceedings of the 13th International Conference on Artificial Intelligence, Expert Systems and Natural Language, Avignon, France.

Parsons, S., Hunter, A., 1998. A review of uncertainty handling formalisms. In: Hunter, A., Parsons, S. (Eds.), Applications of Uncertainty Formalisms, vol. 1455, Lecture Notes in Artificial Intelligence, Springer, Berlin, pp. 8-37.

Pattan, B., 1998. Satellite-Based Global Cellular Communications. McGraw-Hill, New York.

Pope, H., Hardy, Q., 1999. Glitches surface as Iridium phones go to war. Wall St. J., 27 April 1999. B1.

Preist, C., 1998. Economic Agents for Automated Trading. Technical ReportHPL-98-77, Hewlett-Packard Laboratories, Bristol, UK.

Raven, D., 1996. The enculturation of logical practice. Configurations 3, 381-425.

Rhinds, S., 1999. Globalstar expects break-even results in first full year. Wall St. J., 26 February, B5B.

Roberts, J.H., 1998. Marketing approaches to forecasting problems. J. Forecasting 17, 169-174.

Robinson, P.B., 1984. Long term demand for processed meat products in Zimbabwe. Report for the Cold Storage Commission of Zimbabwe, Zimconsult Ltd., Harare, Zimbabwe.

Rogers, E.M., 1983. Diffusion of Innovations, third ed.. The Free Press, New York.

Schwartz, P., 1991. The Art of the Long View. Doubleday, New York.

Stanton, J.L., Chandran, R., Hernandez, S.A., 1982. Marketing research problems in Latin America. J. Market Res. Soc. 24, 124-139. 
Stepick, A., Stepick, C.D., 1990. People in the shadows: survey research among Haitians in Miami. Hum. Organ. 49 (1), 64-77.

Turksen, I.B., Willson, I.A., 1995. A fuzzy set model for market share and preference prediction. Eur. J. Oper. Res. 82, 39-52.

UMTS Forum 1999. The future mobile market: global trends and developments with a focus on Western Europe. Report 8, Universal Mobile Telecommunications Services Forum, London, UK.

Urban, G.L., Hauser, J.R., 1993. Design and Marketing of New Products. Prentice-Hall, Englewood Cliffs, NJ.

Urban, G.L., Hauser, J.R., Roberts, J.H., 1990. Prelaunch forecasting of new automobiles. Manage. Sci. 36 (4), 401-421.

Urban, G.L., Weinberg, B.D., Hauser, J.R., 1996. Premarket forecasting really new products. J. Marketing 60 (1), 47-60.

Walsham, G., 1982. A model of future telecommunications demand. J. Operational Res. Soc. 33, A34.

Wetenhall, R., 1998. Propagation delay - an MSS red herring. Mobile Communications International 25, June. 